\title{
Detecção precoce do câncer de mama em Unidades Básicas de Saúde
}

Early detection of breast cancer in primary care center

Detección precoz de cáncer de mama en unidades básicas de salud

Fabiana Barbosa Barreto Melo ${ }^{1}$ ic https://orcid.org/0000-0001-8977-638X

Elisabeth Níglio de Figueiredo ${ }^{1}$ io https://orcid.org/0000-0001-5939-8306

Marislei Sanches Panobianco ${ }^{2}$ io https://orcid.org/0000-0003-2619-8740

Maria Gaby Rivero de Gutiérrez'11 in https://orcid.org/0000-0003-4189-1594

Anderson da Silva Rosa' ${ }^{1}$ io https:/orcid.org/0000-0003-4683-3107

\section{Como citar:}

Melo FB, Figueiredo EN, Panobianco MS, Gutiérrez MG, Rosa AS. Detecção precoce do câncer de mama em Unidades Básicas de Saúde. Acta Paul Enferm. 2021;34:eAPE02442.

DOI

http://dx.doi.org/10.37689/actaape/2021A002442

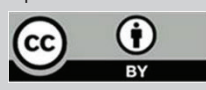

Descritores

Neoplasias da mama; Programas de rastreamento; Atenção primária à saúde; Estratégia saúde da família; Papel do profissional de enfermagem;

Centros de saúde

Keywords

Breast neoplams; Mass screening: Primary health care; Family health strategy; Nurse's role; Health

centers

\section{Descriptores}

Neoplasias de la mama; Tamizaje masivo; Atención primaria de salud; Estrategia de salud familiar; Rol de la enfermeira; Centros de salud

\section{Submetido 20 de Agosto de 2019 \\ Aceito 20 de Agosto de 2020}

Autor correspondente Fabiana Barbosa Barreto Melo E-mail: fabianbs@gmail.com

\section{Resumo}

Objetivo: Analisar as ações para detecção precoce do câncer de mama realizadas por enfermeiros da atenção primária, de acordo com as diferentes configurações de unidades básicas de saúde.

Método: Estudo de corte transversal, desenvolvido em 38 unidades básicas de saúde. As unidades foram selecionadas mediante cálculo de amostra aleatória simples; os critérios de inclusão foram os enfermeiros de ambos os sexos, com tempo mínimo de um ano de atuação na unidade. As diferentes hipóteses cogitadas foram avaliadas por meio de análise bivariada na tabela de contingência, utilizando 0 teste de qui-quadrado ou teste exato de Fischer; todos os testes realizados levaram em consideração um $\alpha$ bidirecional de 0,05 e intervalo de confiança (IC) de 95\%.

Resultado: Dos 133 enfermeiros do estudo, 46,6\% atuavam em unidades básicas da Estratégia Saúde da Família, 31,6\% em unidades mistas e 21,8\% em unidades tradicionais. Houve melhor desempenho para 0 modelo Estratégia Saúde da Família, com resultados estatisticamente significativos para as seguintes ações: investigação dos fatores de risco $(p=<0,001)$; orientação da idade ideal para exame clínico das mamas e a importância de sua realização ( $p=0,002$ e $p=<0,001$ respectivamente); reunião educativa sobre câncer de mama ( $p=<0,001)$; busca ativa de mulheres com laudo suspeito $(p=0,002)$ e encaminhamento à unidade de referência $(p=<0,001)$.

Conclusão: As ações para a detecção precoce do câncer de mama realizadas pelos enfermeiros diferenciamse em relação à configuração da Unidade Básica de Saúde, sendo que as do modelo Estratégia Saúde da Família se aproximam mais das recomendações do Ministério da Saúde.

\section{Abstract}

Objective: To analyze the actions for early detection of breast cancer performed by primary care nurses, according to the different configurations of primary care center.

Method: Cross-sectional study, carried out in a total of 38 primary care center. The centers were selected by calculating a simple random sample; the inclusion criteria were nurses of both genders, with at least one year working in the health center. The different hypotheses considered were evaluated by a bivariate analysis in the contingency table, using the chi-square test or Fisher's exact test; all tests performed considered a bidirectional $\alpha$ of 0.05 and a $95 \%$ confidence interval (Cl).

Result: Out of the 133 nurses in the study, 46.6\% worked in primary care center of the Family Health Strategy, $31.6 \%$ in mixed centers and $21.8 \%$ in traditional ones. There was a better performance for the Family Health Strategy model, with statistically significant results for the following actions: investigation of risk factors $(p=<0.001)$; orientation of the ideal age for clinical breast examination and the importance of its 
performance ( $p=0.002$ and $p=<0.001$ respectively); educational meeting on breast cancer $(p=<0.001)$; active search for women with suspicious medical reports $(p=0.002)$ and referral to the health center $(p=<0.001)$.

Conclusion: The actions for early detection of breast cancer performed by nurses differ in relation to the configuration of the Primary care center, and those from the Family Health Strategy model are closer to the recommendations of the Ministry of Health.

\section{Resumen}

Objetivo: Analizar las acciones para la detección precoz de cáncer de mama realizadas por enfermeros de atención primaria, de acuerdo con las diferentes configuraciones de las unidades básicas de salud.

Métodos: Estudio de corte transversal, llevado a cabo en 38 unidades básicas de salud. Las unidades fueron seleccionadas mediante cálculo de muestra aleatoria simple. Los criterios de inclusión fueron enfermeros de ambos sexos, con un tiempo mínimo de un año de actuación en la unidad. Las diferentes hipótesis consideradas fueron evaluadas mediante el análisis bivariado en la tabla de contingencia, utilizando la prueba $\chi^{2}$ de Pearson o prueba exacta de Fisher. Todas las pruebas realizadas tuvieron en consideración un $\alpha$ bidireccional de 0,05 e intervalo de confianza (IC) de $95 \%$.

Resultado: De los 133 enfermeros del estudio, el 46,6 \% actuaba en unidades básicas de Estrategia Salud de la Familia, el 31,6 \% en unidades mixtas y el 21,8 \% en unidades tradicionales. Se observó un mejor desempeño en el modelo Estrategia Salud de la Familia, con resultados estadísticamente significativos en las siguientes acciones: investigación de los factores de riesgo $(\mathrm{p}=<0,001)$, orientación sobre la edad ideal para examen clínico de las mamas y la importancia de su realización ( $p=0,002$ y $p=<0,001$ respectivamente), reunión educativa sobre cáncer de mama ( $p=<0,001$ ), búsqueda activa de mujeres con resultados sospechosos $(p=0,002)$ y derivación a la unidad de referencia $(p=<0,001)$.

Conclusión: Las acciones para la detección precoz de cáncer de mama realizadas por los enfermeros se diferencian según la configuración de la unidad básica de salud, entre las cuales el modelo Estrategia Salud de la Familia está más cerca de las recomendaciones del Ministerio de Salud.

\section{Introdução}

Na cidade de São Paulo, a municipalização da saúde prevista pelo Sistema Único de Saúde (SUS) se deu tardiamente, em 2001. O Município assumiu a rede de centros de saúde do Estado e incorporou-a à rede de unidades básicas de saúde (UBS) já existentes. Concomitantemente, iniciou a implantação e ampliação da Estratégia Saúde da Família (ESF). ${ }^{(1)}$

Mesmo com a implantação da ESF, as UBS estruturaram-se em dois modelos assistenciais: o Tradicional e a Estratégia Saúde da Família. ${ }^{(1)}$ Ambos os modelos têm os mesmos objetivos de prevenção, promoção, diagnóstico, tratamento e reabilitação. No entanto, o modelo tradicional privilegia o atendimento médico por especialidades básicas, pautado no formato queixa-conduta e, pela própria estrutura do serviço, tem limitada atuação na comunidade e nas ações de promoção e prevenção. ${ }^{(1,2)}$

Já na ESF trabalha-se essencialmente em equipe, valoriza-se o papel do enfermeiro e tem, na figura do agente comunitário de saúde (ACS), maior possibilidade de realizar açóes de promoção, prevenção e de detecção precoce de doenças e agravos. ${ }^{(2)}$ Destacase que o modelo ESF propóe a reorganização da Atenção Primária a Saúde (APS) e a consolidação do SUS, sendo o processo de trabalho ancorado nos princípios da clínica ampliada, na proximidade e no vínculo com as famílias e comunidade. ${ }^{(2)}$
Em algumas UBS, esses modelos coexistem e recebem a denominação de UBS-Mista, portanto, a composição das equipes e a forma de trabalho correspondem aos dois modelos apresentados. ${ }^{(1)}$ Sendo assim, adotou-se neste estudo os termos tipo ou configuração de UBS para se referir aos diferentes arranjos organizacionais desses serviços, uma vez que a UBS Mista em si não configura nenhum modelo de atenção à saúde.

Na APS, independente do modelo assistencial, o enfermeiro amplia sua atuação, devido a autonomia em suas práticas, sua participação nos processos educativos, nos movimentos de organização social, gerenciamento e liderança da equipe, ${ }^{(3)}$ além de ser o principal interlocutor das políticas e programas de saúde pública.

Um dos desafios deste profissional, no contexto da atenção aos principais problemas de saúde da população feminina, tem relação com as açóes direcionadas ao rastreamento e diagnóstico precoce do câncer de mama, ${ }^{(4)}$ independentemente do tipo de UBS em que ele desenvolva suas atividades laborais.

Mundialmente, o câncer de mama apresenta uma incidência de 46,3\% antes dos 75 anos, sendo a primeira causa de morte entre as mulheres. ${ }^{(5)}$ No Brasil, são esperados para o biênio 2018-2019, 59.7000 casos novos desta neoplasia, com risco estimado de 56,33 casos por 100,000 mulheres, ${ }^{(6)}$ constituindo-se, assim, em um problema de saúde 
pública que obtém visibilidade na agenda da política de saúde nacional. ${ }^{(7,8)}$

As atuais diretrizes do Ministério da Saúde (MS) para detecção precoce do câncer de mama definem a APS como principal lócus dessa atuação, ${ }^{(7,8)}$ tendo como estratégias a conscientização da população sobre esta patologia; a identificação dos sinais e sintomas e a mamografia (MMG) bienal para as mulheres entre 50 a 69 anos. ${ }^{(7)}$ Já o autoexame das mamas (AEM) e o exame clínico das mamas (ECM) não são indicados como método de rastreamento. No entanto, recomenda-se que essas açóes devam fazer parte da orientação das mulheres para conhecimento do próprio corpo, bem como da propedêutica de atendimento de enfermeiros e médicos.

Ressalta-se que até meados de 2015 era recomendado ECM anual em todas as mulheres a partir de 40 anos de idade com MMG subsequente na presença de alteraçóes, ${ }^{(8,9)}$ sendo que o instrumento de coleta de dados desse estudo foi elaborado no momento em que essa diretriz ainda estava vigente.

Pesquisas apontam lacunas nas açóes de enfermeiros na detecção precoce do câncer de mama nas UBS. Duas revisóes integrativas identificaram falta de conhecimento técnico científico sobre esta neoplasia ${ }^{(4,10)}$ e um estudo atual destaca a necessidade de investimentos na capacitação profissional e gerenciamento dos serviços de saúde. ${ }^{(11)}$

Assim sendo, este estudo teve por objetivo: analisar as ações de detecção precoce do câncer de mama realizadas por enfermeiros da atenção primária de acordo com as diferentes configuraçôes de Unidades Básicas de Saúde.

\section{Métodos}

Trata-se de um estudo de corte transversal, desenvolvido com enfermeiros que desenvolviam suas atividades laborais em 38 UBS da Coordenadoria de Saúde Sudeste do município de São Paulo. A população estimada do território adstrito é de 2.705.660 habitantes, sendo 1.433 .857 mulheres, das quais 744.452 estão na faixa etária da população-alvo das açôes para o controle do câncer de mama. ${ }^{(12)}$
Os enfermeiros foram selecionados por meio de amostragem aleatória simples, considerando os modelos das 90 UBS existentes em 2011, resultando em 17 UBS-Tradicional, 12 UBS-ESF e nove UBSMista. Foram excluídos aqueles que não atuavam diretamente no atendimento às usuárias e os que atuavam como gerentes. Dessa forma, a amostra foi constituída por 133 enfermeiros, sendo 62 de UBS ESF, 42 de UBS-Mista e 29 de UBS-Tradicional. Destaca-se que a Prefeitura de São Paulo firma contratos de gestão com Organizações Sociais, que são responsáveis por algumas destas UBS.

Utilizou-se um questionário construído e validado por Marques ${ }^{(13)}$ que inclui dados de identificação, capacitação, açóes para o controle do câncer de mama (investigação dos fatores de risco, exame clínico das mamas, mamografia, ultrassonografia e autoexame das mamas) e açóes gerais (uso dos sistemas de informação, reunióes educativas e consulta de enfermagem).

Os enfermeiros foram entrevistados, no período de janeiro a março de 2014, por uma das pesquisadoras deste estudo em área reservada, com agendamento prévio, na UBS e assinaram o termo de consentimento livre e esclarecido. Ao término da coleta, as informaçóes foram transmitidas ao banco de dados hospedado no website Observatório do Câncer de Mama. Foi realizada dupla digitação em 10\% dos questionários e em 10\% das questóes, com 100\% de concordância (Coeficiente de Kappa 1.00).

Adotou-se como variável dependente as açóes de detecçáo precoce do câncer de mama resultantes das informações sobre fatores de risco, ECM, MMG e AEM. Os tipos de UBS constituíram as variáveis independentes.

As variáveis qualitativas são apresentadas como o número de frequência absoluta e relativa. Para a comparaçáo das açóes realizadas e o tipo de UBS utilizou-se o teste de qui-quadrado ou exato de Fisher.

Todos os testes realizados levaram em consideração um $\alpha$ bidirecional de 0,05 e intervalo de confiança (IC) de $95 \%$ e foram realizados com apoio computacional dos softwares IBM SPSS 20 (Statistical Package for the Social Sciences) e Excel $201{ }^{\circledR}$ (Microsoft Office). 
O projeto foi aprovado pelos Comitês de Ética em Pesquisa (CEP) da Universidade Federal de São Paulo (UNIFESP) e da Coordenadoria de Saúde da região Sudeste do município de São Paulo e obedeceu aos preceitos ético-legais para pesquisas com seres humanos, de acordo com a Resolução 466/2012 do Conselho Nacional de Saúde.

\section{Resultados}

Apresentam-se nas linhas das tabelas 1, 2 e 3 o número total de participantes $(n=133)$, enquanto nas colunas, o número de enfermeiros conforme o tipo de UBS, de modo a permitir a comparação dos grupos.

A tabela 1 mostra que a maioria dos enfermeiros das UBS $(64,7 \%)$ atuava há menos de cinco anos na UBS, sendo $81 \%$ nas UBS-Mistas e $67,8 \%$ na UBS-ESF. As UBS-Tradicionais foram as que apresentaram o maior percentual de enfermeiros (31\%) com mais de dez anos de atuação no serviço. No que diz respeito à formação, 81,2\% enfermeiros referiram ter realizado curso de especializaçáo, sendo essa proporção menor entre os que atuavam nas UBS-Tradicionais (69\%).

No que se refere a realização do curso de capacitação sobre as ações preconizadas para o controle do câncer de mama verificou-se maior frequência nas UBS-Mistas $(68,3 \%)$ e ESF (62,3\%) quando comparadas às UBS-Tradicionais (50\%) (Tabela 1$)$.

A indicação anual da periodicidade para realizaçáo da MMG foi mencionada pela maioria dos enfermeiros $(72,9 \%)$, sendo que apenas $12,1 \%$ citaram a periodicidade bienal conforme recomenda a Diretriz do MS. Dos 133 enfermeiros, 6,0\% apontaram a faixa etária igual ou maior que 50 anos para realização da $M M G$, prevalecendo a indicação da faixa etária igual ou maior que 40 anos (61,6\%). Nas UBS-Tradicionais, $27,6 \%$ não recordavam a idade indicada. Quanto ao tempo de espera entre a solicitação da MMG e o retorno do resultado, destacou-se o período de um a três meses de espera nos diferentes arranjos organizacionais de UBS pesquisadas. Contudo, a maior proporção de enfermeiros que não recordava este período foi o das
Tabela 1. Características profissionais de enfermeiros, segundo diferentes tipos de UBS $(n=133)$

\begin{tabular}{|c|c|c|c|c|}
\hline \multirow[b]{2}{*}{ Variáveis } & \multicolumn{3}{|c|}{ Tipos de Unidades Básicas de Saúde } & \multirow[b]{2}{*}{$\begin{array}{l}\text { Total } \\
\mathrm{n}(\%)\end{array}$} \\
\hline & $\begin{array}{l}\text { ESF* }^{*} \\
\mathrm{n}(\%)\end{array}$ & $\begin{array}{c}\text { Tradicional } \\
\mathrm{n}(\%)\end{array}$ & $\begin{array}{c}\text { Mista } \\
n(\%)\end{array}$ & \\
\hline \multicolumn{5}{|c|}{ Tempo de atuação } \\
\hline$<5$ anos & $42(67,8)$ & $10(34,5)$ & $34(81,0)$ & $86(64,7)$ \\
\hline $5-10$ anos & $10(16,1)$ & $10(34,5)$ & $3(7,1)$ & $23(17,3)$ \\
\hline$>10$ anos & $10(16,1)$ & $9(31,0)$ & $5(11,9)$ & $24(18,0)$ \\
\hline Total & $62(100,0)$ & $29(100,0)$ & $42(100,0)$ & $133(100,0)$ \\
\hline \multicolumn{5}{|l|}{ Maior titulação } \\
\hline Bacharel & $7(11,3)$ & $6(20,7)$ & $4(9,5)$ & $17(12,8)$ \\
\hline Especialista & $51(82,3)$ & $20(69,0)$ & $37(88,1)$ & $108(81,2)$ \\
\hline Mestre & $4(6,4)$ & $2(6,9)$ & $1(2,4)$ & $7(5,3)$ \\
\hline Doutor & $0(0,0)$ & $1(3,4)$ & $0(0,0)$ & $1(0,7)$ \\
\hline Total & $62(100,0)$ & $29(100,0)$ & $42(100,0)$ & $133(100,0)$ \\
\hline \multicolumn{5}{|c|}{$\begin{array}{l}\text { Especialista em Saúde da } \\
\text { Família/Saúde Pública/ } \\
\text { Obstetrícia }\end{array}$} \\
\hline Sim & $44(71,0)$ & $13(44,8)$ & $30(71,4)$ & $87(65,4)$ \\
\hline Não & $18(29,0)$ & $16(55,2)$ & $12(28,6)$ & $46(34,6)$ \\
\hline Total & $62(100,0)$ & $29(100,0)$ & $42(100,0)$ & $133(100,0)$ \\
\hline \multicolumn{5}{|c|}{$\begin{array}{l}\text { Capacitação sobre ações } \\
\text { preconizadas pelo MS }\end{array}$} \\
\hline Sim & $38(62,3)$ & $14(50,0)$ & $28(68,3)$ & $80(61,5)$ \\
\hline Não & $23(37,7)$ & $14(50,0)$ & $13(31,7)$ & $50(38,5)$ \\
\hline Total & $61(100,0)$ & $28(100,0)$ & $41(100,0)$ & $130^{\star *}(100,0)$ \\
\hline
\end{tabular}

UBS-Tradicional (24,1\%). Dos 133 enfermeiros, $59(44,4 \%)$ referiram a existência de dificuldades para a execução da MMG. O maior percentual foi nas UBS-ESF $(46,8 \%)$ e as dificuldades mais mencionadas foram o agendamento do exame e a falta da usuária à realização do mesmo. A consulta de enfermagem era realizada por $95,5 \%$ do total de enfermeiros, sendo ligeiramente menor na UBSTradicional (86,2\%). Observa-se ainda que 82,3\% e $67,5 \%$ dos enfermeiros das UBS-ESF e Mista realizaram entre 10 a 20 consultas por dia respectivamente; enquanto que na UBS-Tradicional 67\% dos enfermeiros realizavam menos de 10 consultas por dia (Tabela 2).

Quanto a realização da investigação dos fatores de risco evidenciou-se diferença estatisticamente significativa entre os respondentes dos diferentes modelos de UBS $(p=<0,001)$, uma vez que todos os enfermeiros da UBS-ESF referiram realizar esta ação, quando comparados aos $62,1 \%$ da UBSTradicional. A realização do ECM fazia parte da rotina de $93,5 \%$ dos enfermeiros da UBS-ESF, de 95,2\% da UBS-Mistas e de 65,5\% dos enfermeiros da UBS-Tradicional. Em relação à realização de reunião educativa, observou-se maior propor- 
Tabela 2. Ações realizadas por enfermeiros dos diferentes tipos de UBS para a detecção precoce do câncer de mama $(n=133)$

\begin{tabular}{|c|c|c|c|c|}
\hline \multirow[b]{2}{*}{ Variáveis } & \multicolumn{3}{|c|}{ Tipos de Unidades Básicas de Saúde } & \multirow[b]{2}{*}{$\begin{array}{l}\text { Total } \\
\mathrm{n}(\%)\end{array}$} \\
\hline & $\begin{array}{l}\mathrm{ESF}^{*} \\
\mathrm{n}(\%)\end{array}$ & $\begin{array}{c}\text { Tradicional } \\
\mathrm{n}(\%)\end{array}$ & $\begin{array}{l}\text { Mista } \\
\mathrm{n}(\%)\end{array}$ & \\
\hline \multicolumn{5}{|l|}{$\begin{array}{l}\text { Período indicado da realização } \\
\text { da MMG }\end{array}$} \\
\hline Anual & $47(75,8)$ & $13(44,9)$ & $37(88,1)$ & $97(72,9)$ \\
\hline Bienal & $8(12,9)$ & $6(20,7)$ & $2(4,8)$ & $16(12,1)$ \\
\hline $\begin{array}{l}\text { Não há estabelecimento de } \\
\text { intervalo }\end{array}$ & $1(1,6)$ & $0(0,0)$ & $0(0,0)$ & $1(0,7)$ \\
\hline Outro período & $6(9,7)$ & $10(34,4)$ & $3(7,1)$ & $19(14,3)$ \\
\hline Total & $62(100,0)$ & $42(100,0)$ & $42(100,0)$ & $133(100,0)$ \\
\hline \multicolumn{5}{|l|}{$\begin{array}{l}\text { Faixa etária para realizar a } 1^{\circ} \\
\text { MMG }\end{array}$} \\
\hline$<35$ anos & $1(1,6)$ & $2(6,9)$ & $0(0,0)$ & $3(2,3)$ \\
\hline $35-39$ & $19(30,6)$ & $5(17,2)$ & $6(14,3)$ & $30(22,6)$ \\
\hline$\geq 40$ anos & $37(59,7)$ & $12(41,4)$ & $33(78,6)$ & $82(61,6)$ \\
\hline$\geq 50$ anos & $3(4,9)$ & $2(6,9)$ & $3(7,1)$ & $8(6,0)$ \\
\hline Sem restrição etária & $2(3,2)$ & $0(0,0)$ & $0(0,0)$ & $2(1,5)$ \\
\hline Não sabe & $0(0,0)$ & $8(27,6)$ & $0(0,0)$ & $8(6,0)$ \\
\hline Total & $62(100,0)$ & $29(100,0)$ & $42(100,0)$ & $133(100,0)$ \\
\hline \multicolumn{5}{|l|}{$\begin{array}{l}\text { Tempo de espera entre a } \\
\text { solicitação de MMG e o retorno } \\
\text { do resultado }\end{array}$} \\
\hline$<1$ mes & $0(0,0)$ & $3(10,4)$ & $3(7,1)$ & $6(4,5)$ \\
\hline $1-3$ meses & $48(77,4)$ & $15(51,7)$ & $28(66,7)$ & $91(68,4)$ \\
\hline$>3$ a 6 meses & $8(12,9)$ & $3(10,4)$ & $6(14,3)$ & $17(12,8)$ \\
\hline Outro período & $2(3,2)$ & $1(3,4)$ & $0(0,0)$ & $3(2,3)$ \\
\hline Não retorna & $0(0,0)$ & $0(0,0)$ & $1(2,4)$ & $1(0,7)$ \\
\hline Não recorda & $4(6,5)$ & $7(24,1)$ & $4(9,5)$ & $15(11,3)$ \\
\hline Total & $62(100,0)$ & $29(100,0)$ & $42(100,0)$ & $133(100,0)$ \\
\hline \multicolumn{5}{|l|}{$\begin{array}{l}\text { Dificuldades para execução da } \\
\text { MMG }\end{array}$} \\
\hline Sim & $29(46,8)$ & $11(37,9)$ & $19(45,3)$ & $59(44,4)$ \\
\hline Não & $32(51,6)$ & $13(44,9)$ & $20(47,6)$ & $65(48,9)$ \\
\hline Não sabe & $1(1,6)$ & $5(17,2)$ & $3(7,1)$ & $9(6,7)$ \\
\hline Total & $62(100,0)$ & $29(100,0)$ & $42(100,0)$ & $133(100,0)$ \\
\hline \multicolumn{5}{|l|}{$\begin{array}{l}\text { Fator que dificulta a execução } \\
\text { da MMG }{ }^{\star \star}\end{array}$} \\
\hline Agendamento & $20(44,4)$ & $9(20,0)$ & $16(35,6)$ & $45(100,0)$ \\
\hline $\begin{array}{l}\text { Falta de técnico para realizar } \\
\text { a MMG }\end{array}$ & $2(50,0)$ & $1(25,0)$ & $1(25,0)$ & $4(100,0)$ \\
\hline Falta mamógrafo & $6(46,1)$ & $4(30,7)$ & $3(23,1)$ & $13(100,0)$ \\
\hline Falta da usuária & $12(63,1)$ & $2(10,6)$ & $5(26,3)$ & $19(100,0)$ \\
\hline \multicolumn{5}{|l|}{$\begin{array}{l}\text { Realização das consultas de } \\
\text { enfermagem }\end{array}$} \\
\hline Sim & $62(100,0)$ & $25(86,2)$ & $40(95,2)$ & $127(95,5)$ \\
\hline Não & $0(0,0)$ & $4(13,8)$ & $2(4,8)$ & $6(4,5)$ \\
\hline Total & $62(100,0)$ & $29(100,0)$ & $42(100,0)$ & $133(100,0)$ \\
\hline \multicolumn{5}{|l|}{$\begin{array}{l}\text { Número de consultas de } \\
\text { enfermagem realizadas por dia }{ }^{\star \star \star}\end{array}$} \\
\hline$<10$ & $11(17,7)$ & $17(68,0)$ & $13(32,5)$ & $0(0,0)$ \\
\hline 10-20 consultas & $51(82,3)$ & $8(32,0)$ & $27(67,5)$ & $0(0,0)$ \\
\hline Total & $62(100,0)$ & $25(100,0)$ & $40(100,0)$ & $127(100,0)$ \\
\hline \multicolumn{5}{|l|}{$\begin{array}{l}\text { Motivo de NÃO realizar a consulta } \\
\text { de enfermagem }{ }^{\star \star}\end{array}$} \\
\hline Falta de tempo & $0(0,0)$ & $3(100,0)$ & $0(0,0)$ & $3(100,0)$ \\
\hline Sobrecarga de trabalho & $0(0,0)$ & $4(80,0$ & $1(20,0)$ & $5(100,0)$ \\
\hline Falta de local apropriado & $0(0,0)$ & $1(50,0)$ & $1(50,0)$ & $2(100,0)$ \\
\hline
\end{tabular}

*ESF - Estratégia Saúde da Familia; * *Questão com possibilidade de mais de uma resposta; * ** missing date $=6$ ção nas UBS-ESF (79,0\%), comparado às UBSTradicionais $(35,7 \%)$. Essas diferenças foram estatisticamente significativas. A busca ativa das mulheres com laudo de MMG suspeito de malignidade foi mencionada por mais da metade dos enfermeiros das diferentes configuraçóes organizacionais, com maior proporção para UBS-ESF $(p=0,002)$, quando comparado aos outros tipos de UBS. O encaminhamento à unidade de referência das mulheres com resultado de MMG suspeito para a neoplasia maligna foi referido por $50,0 \%$ dos enfermeiros da UBSESF, valor superior e estatisticamente significativo quando comparado aos enfermeiros das outras configuraçóes de UBS $(p=<0,001)$ (Tabela 3).

\section{Discussão}

As diretrizes do MS para o controle do câncer de mama têm sido revisadas em consonância com as evidências científicas e estabelecem um conjunto de açôes para adoção de hábitos saudáveis, rastreamento, diagnóstico precoce, tratamento, reabilitação e cuidados paliativos. Propóem uma política transversal, integrada e intersetorial que irá dialogar com as mais diversas áreas para promover longevidade com qualidade de vida para a população feminina brasileira. Foca-se na realização de ações de detecção precoce do câncer de mama na Atenção Básica e atribui aos enfermeiros a responsabilidade de gestão e execução de grande parte dessas açóes.

No Brasil, a Atençáo Básica tem como principal modelo de atenção a Estratégia Saúde da Família coexistindo com outros arranjos organizacionais para o funcionamento das UBS. ${ }^{(2)}$ Apesar de certa flexibilidade na estruturação desses serviços, as diretrizes para o controle do câncer de mama são as mesmas. $\mathrm{Na}$ literatura é incipiente a abordagem sobre as açóes de detecção precoce do câncer de mama, sobretudo comparando os resultados obtidos nesses diferentes tipos de UBS.

Os resultados deste estudo mostram uma maior proporção de enfermeiros atuando em UBS-ESF, assim como nas UBS-Mistas, onde a maioria deles trabalhavam na lógica da ESF. Tal resultado corrobora as modificaçóes na estrutura da APS no município de 
Tabela 3. Associação entre as variáveis relacionadas à detecção precoce do câncer de mama de acordo com os diferentes tipos de UBS $(n=133)$

\begin{tabular}{|c|c|c|c|c|c|c|c|c|}
\hline \multirow{4}{*}{ Variáveis } & \multicolumn{6}{|c|}{ Tipos de Unidades Básicas de Saúde } & \multirow{4}{*}{$\begin{array}{c}\text { Total } \\
\mathrm{n}\end{array}$} & \multirow{4}{*}{$p$-value } \\
\hline & \multicolumn{2}{|c|}{$\operatorname{ESF}^{\star}(n=62)$} & \multicolumn{2}{|c|}{ Tradicional $(\mathrm{n}=29)$} & \multicolumn{2}{|c|}{ Mista $(\mathrm{n}=42)$} & & \\
\hline & $\operatorname{Sim}$ & Não & Sim & Não & Sim & Não & & \\
\hline & $\mathrm{n}(\%)$ & $\mathrm{n}(\%)$ & $\mathrm{n}(\%)$ & $\mathrm{n}(\%)$ & $n(\%)$ & $\mathrm{n}(\%)$ & & \\
\hline Investigação dos fatores de risco & $62(100,0)$ & $0(0,0)$ & $18(62,1)$ & $11(37,9)$ & $40(95,2)$ & $2(4,8)$ & 133 & $<0,001$ \\
\hline Realização do ECM & $58(93,5)$ & $4(6,5)$ & $19(65,5)$ & $10(34,5)$ & $40(95,2)$ & $2(4,8)$ & 133 & $<0,001$ \\
\hline Orientação sobre a idade de início do ECM & $47(75,8)$ & $15(24,2)$ & $12(41,4)$ & $17(58,6)$ & $33(78,6)$ & $9(21,4)$ & 133 & 0,002 \\
\hline Existência de dificuldade na realização do ECM & $32(51,6)$ & $30(48,4)$ & $19(65,5)$ & $10(34,5)$ & $17(40,5)$ & $25(59,5)$ & 133 & 0,117 \\
\hline Realização de reunião educativa & $49(79,0)$ & $13(21,0)$ & $10(35,7)$ & $18(64,3)$ & $29(69,1)$ & $13(30,9)$ & $132^{\star *}$ & $<0,001$ \\
\hline Busca ativa das mulheres com laudo suspeito de MMG & $55(88,7)$ & $7(11,3)$ & $17(58,6)$ & $12(41,4)$ & $28(66,7)$ & $14(33,3)$ & 133 & 0,002 \\
\hline Busca ativa das mulheres que faltaram à MMG & $19(30,6)$ & $43(69,4)$ & $4(13,8)$ & $25(86,2)$ & $14(33,3)$ & $28(66,7)$ & 133 & 0,147 \\
\hline Encaminhamento a unidade de referência & $31(50,0)$ & $31(50,0)$ & $5(17,2)$ & $24(82,8)$ & $7(16,7)$ & $35(83,3)$ & 133 & $<0,001$ \\
\hline Especialista em Saúde da Família/ Saúde Pública/ Obstetrícia & $44(71,0)$ & $18(29,0)$ & $13(44,8)$ & $16(55,2)$ & $30(71,4)$ & $12(28,6)$ & 133 & 0,031 \\
\hline
\end{tabular}

Teste exato de Fischer; *ESF - Estratégia Saúde da Família; **missing date $=1 ; p$-value $=0,05$

São Paulo, uma vez que houve ampliação da cobertura desse modelo de UBS, passando de 22,49\% em 2005 para $38,7 \%$ até março de $2019^{(14)}$, fato que também pode explicar a maior proporção de enfermeiros com menos de cinco anos de atuação nas UBS-ESF e Mista.

Com relação à formação dos enfermeiros, a maioria referiu possuir título de especialista com destaque aos das UBS-ESF. Pode-se refletir que a formação dos profissionais para a APS tem sido objeto de preocupação, sugerindo que a procura pela especialização pode estar relacionada às exigências do mercado de trabalho. ${ }^{(15)}$

Também houve crescimento substancial das atribuiçóes do enfermeiro na rede de atenção básica, contudo, conforme o estudo de Melo, ${ }^{(15)}$ há necessidade de maior investimento institucional no processo de educação permanente desses profissionais quanto a sua atuação na APS. Além disso, é preciso modernizar as estratégias didáticas utilizadas e os conteúdos ministrados, visando sua real contribuição para o sistema de saúde centrado neste modelo de atenção. ${ }^{(16)}$

Ressalta-se que existe certa incongruência entre as açóes de promoção de saúde, prevenção de doenças e agravos a serem desenvolvidas pelos enfermeiros da APS e as normas de acompanhamento do trabalho desses profissionais estabelecidas no município de São Paulo. Há a preponderância de consultas individuais e açôes programáticas na composição das metas de produção que compóem os critérios de avaliação e monitoramento dos contratos de gestão firmados entre a Prefeitura e as Organizaçóes Sociais responsáveis pelas UBS. ${ }^{(17)}$ Em contrapartida, as açóes de promoção e prevenção demandam tempo e persistência, pois envolvem a criação de vínculo, elemento chave desse processo e um dos pressupostos que regem a APS.

A consulta de enfermagem é o principal indicador de produção para os enfermeiros e, neste estudo, quase a totalidade dos enfermeiros relatou realizá-la, sendo esse percentual 10\% inferior na UBS Tradicional. Ressalta-se que a pressão para o cumprimento das metas pode ser menor para os enfermeiros da UBSTradicional contratados pelo regime estatutário. Assim, faz-se necessário considerar a influência de diferentes vínculos trabalhistas e formas de acompanhamento na qualidade das açóes realizadas na APS.

Apesar de a avaliação do trabalho da ESF ser pautada por metas, a rigidez com que esse processo tem sido construído, contribui com a sobrecarga dos profissionais. Tal situação gera distorçôes no processo de trabalho das equipes, sobrevalorizando o cumprimento de indicadores quantitativos, condiçáo que tem sido apontada como fator limitante para a qualidade do trabalho do enfermeiro em diferentes programas e atribuiçóes. ${ }^{(18,19)}$

Outro aspecto observado diz respeito ao Instrumento Normativo para Monitoramento Técnico, Administrativo e Financeiro dos convênios da Estratégia Saúde da Família do Município de São Paulo que estabelece como indicador para acompanhamento e avaliação no eixo saúde da mulher, apenas a Proporção de Gestantes Acompanhadas. ${ }^{(17)}$ Nesse sentido, as açôes de detecção precoce do câncer de mama, apesar de incentivadas pelo MS em diferentes documentos, ${ }^{(7-9)}$ podem ser negligenciadas, à medida que competem com outras metas quantitativas de produção.

Assim, o atendimento à saúde integral das mulheres é um desafio que precisa ser superado com respon- 
sabilidade. Faz-se necessário o cumprimento das recomendaçốes do $\mathrm{MS}^{(20)}$ tanto pelos enfermeiros e equipes de saúde, quanto pelos serviços que compóem o SUS. Tềm-se evidências suficientes para justificar o investimento na detecção precoce do câncer de mama, ${ }^{(21)}$ considerada uma doença de bom prognóstico, desde que diagnosticada e tratada em tempo. ${ }^{(22)}$

Com relação ao período e a faixa etária para a realização da MMG, observa-se a não conformidade das açôes para a detecçáo precoce desta neoplasia segundo a recomendação do MS. ${ }^{(7)}$ Pondera-se que o rastreamento são testes realizados em indivíduos assintomáticos ${ }^{(7)} \mathrm{e}$ que as exigências para a sua indicação devem ser seguidas com rigor, uma vez que os danos iatrogênicos nem sempre estão contrabalançados com a compensação da cura, controle ou alívio do adoecimento sentido. Devese evitar que pessoas que se percebem saudáveis tenham dimensóes da sua saúde abalada, indefinidamente, devido às intervençóes de rastreamento mal realizadas. ${ }^{(23)}$

Também cabe ressaltar que no momento da coleta de dados, a solicitaçáo de MMG era responsabilidade exclusiva dos médicos no município de São Paulo, cuja conduta pode estar sendo influenciada pelas recomendaçóes ${ }^{(24)}$ do Colégio Brasileiro de Radiologia e Diagnóstico por Imagem (CBR), da Sociedade Brasileira de Mastologia (SBM) e da Federação Brasileira das Associaçóes de Ginecologia e Obstetrícia (FEBRASGO), que indicam a realização da MMG para todas as mulheres na faixa etária de 40 a 69 anos, com periodicidade anual, divergindo do Documento de Consenso $^{(9)}$ e das novas Diretrizes ${ }^{(7)}$ do MS que recomendam o rastreamento por MMG para as mulheres com idade entre 50 a 69 anos, com intervalo de dois anos entre os exames.

Esse cenário de divergências pode interferir nas açóes realizadas pelos enfermeiros e evidencia a necessidade de que se estabeleça um consenso no município para que a APS coordene as redes de atenção à saúde. Ressalta-se que o Conselho Regional de Enfermagem de São Paulo-Coren, ${ }^{(25)}$ juntamente com a Secretária de Saúde, ${ }^{(26)}$ autorizam os enfermeiros a solicitar MMG na rede de Atenção Básica.

Em relaçáo às mulheres com laudo de MMG suspeito de malignidade, a maior parte dos enfermeiros referiram realizar a busca ativa, destacando-se os enfermeiros da UBS-ESF, com diferença estatística sig- nificativa em relação às demais configuraçóes de UBS. Quanto a busca ativa das faltosas, esta foi referida por um número reduzido de enfermeiros atuantes nos três tipos de UBS. Este resultado corrobora os estudos de Sousa ${ }^{(27)}$ e de Teixeira, ${ }^{(28)}$ que destacam a deficiência na busca ativa das faltosas e apontam necessidade de maior controle, por parte das UBS, das mulheres nas diferentes possibilidades de itinerários terapêuticos.

Destaca-se que açóes de busca ativa são de suma importância tanto para o rastreamento, quanto para a elucidação diagnóstica e início do tratamento. Sua não execução denota falhas da APS para atuar como coordenadora da assistência à saúde dos usuários adscritos. ${ }^{(4)}$

O encaminhamento das mulheres com resultado do exame de MMG alterado para as unidades de referência merece atenção, pois pouco mais de um quarto dos enfermeiros relatou realizá-lo. Tal resultado pode ser explicado pelos fluxos de encaminhamento estabelecidos no município para o câncer de mama que centraliza essa função nos profissionais médicos. Tal determinação impede o pleno cumprimento de atividades que podem ser executadas por enfermeiro na $\operatorname{APS}^{(26,29)}$ e nos serviços onde não houver facilidade de acesso ao médico, existindo o risco de atraso no encaminhamento e, consequentemente, na qualificação do diagnóstico e início do tratamento.

Não foram encontrados estudos relacionados ao câncer de mama que comparassem os modelos de atenção ESF e tradicional. Em contrapartida, essa comparação tem sido abordada em estudos com diferentes enfoques e apontam para a superioridade do modelo ESF com relação ao modelo tradicional na realização de açôes específicas, ${ }^{(30)}$ da organização do serviço voltados à política nacional de atenção básica-PNAB, ${ }^{(31)}$ da capacitação dos profissionais, ${ }^{(30)}$ do melhor desempenho e resolubilidade, ${ }^{(32)}$ bem como maior satisfação do usuário.

$\mathrm{Na}$ presente investigação, ao se cotejar os resultados obtidos da relação entre a realização das ações para a detecção precoce do câncer de mama e o tipo de UBS, houve melhor desempenho para as UBS-ESF, com resultados estatisticamente significativos para as variáveis, investigação dos fatores de risco, reuniāo educativa sobre a temática, profissionais especialistas em Saúde da Família/Saúde Pública/Obstetrícia, bem como enfermeiros com curso de capacitação. 
Esses achados permitem levantar a hipótese de que os enfermeiros da UBS-ESF tiveram resultados mais favoráveis possivelmente pelo fato deste modelo de atenção assumir, de forma mais efetiva, o papel de reorientar a prática assistencial, agindo como centro ordenador das redes de atenção à saúde do SUS.

Em contraposição, o estudo de Barbosa ${ }^{(33)}$ observou a falta de familiaridade com os fatores de alto risco e das indicaçôes de realização da MMG com 80 enfermeiros da ESF em São Luiz - Maranhão.

Os resultados apresentados permitem uma avaliação das açóes para detecção precoce do câncer de mama em parte da rede de APS do município de São Paulo. A redução da mortalidade por essa neoplasia depende de um conjunto de outras açóes coordenadas do Sistema de Saúde e configura uma dimensão importante do cuidado integral à saúde das mulheres brasileiras.

Vale destacar que a implantação do modelo ESF nos grandes centros urbanos é complexa, fato que pode ocasionar a coexistência dos tipos de UBSESF, Mista e Tradicional como mostra neste estudo.

Salienta-se também que a melhora nos indicadores de morbimortalidade do câncer de mama, almejados pelas diretrizes do Ministério da Saúde, exige comprometimento na qualificação dos processos de trabalho das práticas clínicas e de gestão do SUS.

Considera-se limitação do estudo a não realização da análise em separado dos resultados correspondentes aos enfermeiros das UBS-Mista, uma vez que dos 42 enfermeiros vinculados a essa configuração de UBS, apenas oito trabalhavam no modelo tradicional. Dado a esse desequilíbrio e ao risco de viés na análise dos resultados, focamos nossa discussão na comparação das ações realizadas pelos enfermeiros dos modelos ESF e tradicional.

\section{Conclusão}

Ao analisar as ações realizadas pelos enfermeiros da APS da região Sudeste do município de São Paulo para a detecção precoce do câncer de mama, a partir das diferentes configuraçóes de UBS, pode-se concluir que todas apresentam inadequaçóes. Não há realização de busca ativa de todas as pacientes com laudos mamográficos alterados, há equívocos na indicação da periodicidade da MMG, na investigação dos fatores de risco e no encaminhamento à unidade de referência. Porém, estas inadequaçóes diferenciam-se de acordo com os diferentes modelos de atenção à saúde adotados. As UBS-ESF tendem a ser mais coerentes com as recomendações da Política Nacional de Atenção Básica e do MS para detecção precoce do câncer de mama do que os demais tipos de UBS. Este estudo também permitiu afirmar que há necessidade de que sejam operados ajustes na execução dessas ações, a fim de adequá-las às diretrizes preconizadas pelo MS e não somente seguir o que é preconizado pela prefeitura de São Paulo para o controle desse agravo, assim como diminuir as disparidades entre os serviços da APS. Dessa forma, novos estudos do tipo quase experimental são necessários no âmbito do processo de intervenção, por meio de metodologias qualitativas e quantitativas que contribuam para a ampliação e aprofundamento deste tema, com a perspectiva de haver mudanças comportamentais e culturais do profissional e da política de saúde para a assistência.

\section{Agradecimentos}

A pesquisa recebeu fomento do Conselho Nacional de Desenvolvimento Científico e Tecnológico - CNPQ.

\section{Colaborações}

Melo FBB, Figueiredo EN, Gutiérrez MGR e Rosa AS, participaram na concepção do estudo, análise e interpretação dos dados, redação do artigo, revisão crítica do conteúdo e aprovação da versão final a ser publicada. Panobianco MS participou da revisão crítica do conteúdo e aprovação da versão final a ser publicada.

\section{Referências}

1. Marques CA, Figueiredo EN, Gutiérrez MG, Cassenote AJ. Breast cancer control in primary health care: challenges in building a sampling plan. Rev APS. 2014;17(2):263-7. 
2. Brasil. Ministério da Saúde. Secretaria de Atenção à Saúde. Departamento de Atenção Básica. Política Nacional de Atenção Básica [Internet]. Brasília, 2012 [citado 2019 Jun 23]. Disponível em: http://189.28.128.100/dab/docs/publicacoes/geral/pnab.pdf

3. Galavote HS, Zandonade E, Garcia AC, Freitas PS, Seidl H, Contarato PC, et al. The nurse's work in primary health care. Esc Anna Nery. 2016;20(1):90-8.

4. Cavalcante SA, Silva FB, Marques CA, Figueiredo EN, Gutiérrez MG. Nurse actions towards breast cancer screening and early diagnosis in Brazil. Rev Bras Cancerol. 2013;59(3):459-66.

5. Ferlay J, Colombet M, Soerjomataram I, Mathers C, Parkin DM, Piñeros M, Znaor A, Bray F. Estimating the global cancer incidence and mortality in 2018: GLOBOCAN sources and methods. Int J Cancer. $2019 ; 144(8): 1941-53$.

6. Instituto Nacional de Câncer José Alencar Gomes da Silva (INCA). Coordenação de Prevenção e Vigilância Estimativa 2018-2019: incidência de câncer no Brasil. Rio de Janeiro: INCA; 2017.

7. Instituto Nacional de Câncer José Alencar Gomes da Silva (INCA). Diretrizes para a detecção precoce do câncer de mama no Brasil. Rio de Janeiro: INCA; 2015.

8. Brasil. Ministério da Saúde. Secretaria de Atenção à Saúde. Departamento de Atenção Básica. Controle dos cânceres do colo do útero e da mama [Internet]. Brasília (DF): Ministério da Saúde; 2013. (Cadernos de Atenção Básica n. 13) (Série A. Normas e Manuais Técnicos). [citado 2019 Jun 18]. Disponível em: https://aps.saude.gov.br/biblioteca/index

9. Instituto Nacional de Câncer José Alencar Gomes da Silva (INCA). Controle do câncer de mama. Documento de Consenso. Brasília (DF): INCA; 2004.

10. Ohl IC, Ohl Rl, Chavaglia SR, Goldman RE. Public actions for control of breast cancer in Brazil: integrative review. Rev Bras Enferm. 2016;69(4):793-803.

11. Moraes DC, Almeida AM, Figueiredo EN, Loyola EA, Panobianco MS. Opportunistic screening actions for breast cancer performed by nurses working in primary health care. Rev Esc Enferm USP. 2016;50(1):14-21.

12. São Paulo (SP). Secretaria Municipal da Saúde. Coordenação de Epidemiologia e Informação - CEInfo. Boletim CEInfo Saúde em Dados Ano XVIII, nº 18, Junho 2019. São Paulo (SP). [Internet]. São Paulo; 2019. [citado 2019 Out 28]. Disponível em: https://www.prefeitura. sp.gov.br/cidade/secretarias/upload/saude/arquivos/publicacoes/ Boletim_CEInfo_Dados_2019.pdf

13. Marques CA, Figueiredo EN, Gutiérrez MG. Validation of an instrument to identify actions for screening and detection of breast cancer. Acta Paul Enferm. 2015;28(2):183-9.

14. Brasil. Ministério da Saíde. E-Gestor: Departamento de Atenção Básica [Internet]. Brasília (DF): Ministério da Saúde; 2017. [citado 2019 Jun 18]. Disponível em: https://egestorab.saude.gov.br/ paginas/acessoPublico/relatorios/relHistoricoCoberturaAB. xhtml;jsessionid=t8z1kl0DpiJkIKvp6Sn5TeW9

15. Melo FB, Marques CA, Rosa AD, Fiqueiredo EN, Gutiérrez MG. Actions of nurses in early detection of breast cancer. Rev Bras Enferm. 2017;70(6):1119-28.

16. Arantes LJ, Shimizu HE, Merchán-Hamann E. The benefits and challenges of the Family Health Strategy in Brazilian Primary Health care: a literature review. Cien Saude Colet. 2016;21(5):1499-510.

17. São Paulo. Secretaria Municipal de saúde. Instrumento normativo para monitoramento técnico, administrativo e financeiro dos convênios da Estratégia Saúde da Família no âmbito do município de São Paulo. [Internet]. São Paulo; Coordenação de Atenção Básica; 2009. [citado 2019 Jun 17]. Disponível em: http://www.prefeitura.sp.gov.br/cidade/ secretarias/upload/saude/arquivos/esf/instrumento_normativo_ monitoramento_tecnico.pdf
18. Fernandes LC, Machado RZ, Anschau GO. Management of health services: developed competencies and difficulties found in basic attention. Cien Saude Coletiva. 2009;14 Suppl 1:1541-52.

19. Matuda CG, Pinto NR, Martins CL, Frazão P. Interprofessional collaboration in the Family Health Strategy: implications for the provision of care and work management. Cien Saude Coletiva. 2015;20(8):2511-21.

20. Zapponi AL, Tocantins FR, Vargens OM. The nurse in the early detection of breast cancer in primary health care. Rev Enferm UERJ. 2015;23(1):33-8.

21. Freitas C, Tura LF, Costa N, Duarte J. A population-based breast cancer screening programme: conducting a comprehensive survey to explore adherence determinants. Eur J Cancer Care (Engl). 2012;21(3):349-59.

22. Gebrim LH, Quadros LG. Breast cancer screening in Brazil. Rev Bras Ginecol Obstet. 2006;28(6):319-23.

23. Norman $A H$, Tesser CD. Access to healthcare in the Family Health Strategy: balance between same day access and prevention/health promotion. Saúde Soc São Paulo. 2015;24(1):165-79.

24. Urban LA, Schaefer MB, Duarte DL, Santos RP, Maranhão NM, Kefalas $\mathrm{AL}$, et al. Recommendations of Colégio Brasileiro de Radiologia e Diagnóstico por Imagem, Sociedade Brasileira de Mastologia, and Federação Brasileira das Associações de Ginecologia e Obstetrícia for imaging screening for breast cancer. Radiol Bras. 2012;45(6):334-9.

25. Conselho Regional de Enfermagem de São Paulo (COREN). Parecer CORENSP 028/2011. Solicitação de Mamografia pelo Enfermeiro [Internet]. São Paulo: COREN; 2011. [citado 2019 Jun 23]. Disponível em: http://portal. coren-sp.gov.br/sites/default/files/parecer_coren_sp_2011_28.pdf

26. São Paulo (Cidade). Secretaria da Saúde. Manual técnico: saúde da mulher nas Unidades Básicas de Saúde / Secretaria da Saúde, Coordenação da Atenção Básica/Estratégia Saúde da Família. - 4ª ed. - (Série Enfermagem). São Paulo: SMS, 2015. [citado 2019 Jun 18]. Disponível em: http://www.prefeitura.sp.gov.br/cidade/secretarias/ upload/saude/arquivos/legislacao/SaudedaMulher23092015.pdf

27. Sousa MM, Figueiredo EN, Gonçalves VC, Gutiérrez MG. Women with mammographic changes: journey on a basic health unit. Rev Enferm UFPE Online. 2017;11(3):1244-54.

28. Teixeira MS, Goldman RE. Gonçalves VCS, Gutiérrez MGR, Figueiredo EN. Primary care nurses' role in the control of breast cancer. Acta Paul Enferm. 2017;30(1):1-7

29. Conselho Regional de Enfermagem de São Paulo (COREN). Parecer COREN-SP 50/2013. Ementa: Encaminhamento de paciente para outros profissionais pelo Enfermeiro [Internet]. São Paulo: COREN; 2013. [citado 2019 Jun 23]. Disponível em: http://portal.coren-sp.gov. br/sites/default/files/parecer_coren_sp_2013_50.pdf

30. Vasquez J, Dumith SC, Susin LR. Maternal breastfeeding: a comparative study of knowledge and management among Family Health Strategy professionals and those using the traditional model. Rev Bras Saúde Mater Infant. 2015;15(2):181-92.

31. Chomatas E, Vigo A, Marty I, Hauser L, Harzheim E. Evaluation of the presence and extension of the attributes of primary care in Curitiba. Rev Bras Med Fam Comunidade. 2013;8(29):294-303.

32. Facchini LA, Piccini RX, Tomasi E, Thumé E, Silveira DS, Siqueira FV, Rodrigues MA. Performance of the PSF in the Brazilian South and Northeast: institutional and epidemiological Assessment of Primary Health Care. Cien Saude Coletiva. 2006;11(3):669-81.

33. Barbosa YC, Rabêlo PP, Aguiar MI, Azevedo PR, Cortês LS. Early detection of breast cancer: how do the nurses in Primary Health Care perform? Rev APS. 2018;21(3):375-86. 